\title{
Accuracy of EGN model in ultra- wideband optical fiber communication systems
}

Liu, Zheng, Xu, Tianhua, Ding, Jianzheng, Zhang, Yunfan, $\mathrm{Li}$, Mupeng, et al.

Zheng Liu, Tianhua Xu, Jianzheng Ding, Yunfan Zhang, Mupeng Li, Tongyang Xu, Tiegen Liu, "Accuracy of EGN model in ultra-wideband optical fiber communication systems," Proc. SPIE 11891, Semiconductor Lasers and Applications XI, 1189106 (9 October 2021); doi: 10.1117/12.2601076

SPIE. Event: SPIE/COS Photonics Asia, 2021, Nantong, Jiangsu, China 


\title{
Accuracy of EGN Model in Ultra-Wideband Optical Fiber Communication Systems
}

\author{
Zheng Liu ${ }^{\mathrm{a}}$, Tianhua Xu ${ }^{\mathrm{a}, \mathrm{b}, \mathrm{c}}$, Jiazheng Ding ${ }^{\mathrm{a}}$, Yunfan Zhang ${ }^{\mathrm{a}}$, Mupeng $\mathrm{Li}^{\mathrm{a}}$, Tongyang $\mathrm{Xu}^{\mathrm{c}}$, and \\ Tiegen $\mathrm{Liu}^{\mathrm{a}}$ \\ aTianjin University, 300072, Tianjin, China \\ ${ }^{\mathrm{b}}$ University of Warwick, CV4 7AL, Coventry, United Kingdom \\ ${ }^{\mathrm{c}}$ University College London, WCIE 6BT, London, United Kingdom
}

\begin{abstract}
The efficient and accurate evaluation of the transmission performance of high-capacity optical communication systems has always attracted significant research attentions. The enhanced Gaussian noise (EGN) model is considered as an excellent solution to predict the system performance taking into account linear and nonlinear transmission impairments. Since the conventional form of the EGN model is complicated and intractable for a fast computation, the closed-form simplification has been regarded as a direction to significantly reduce the computational complexity. However, the accuracy of such a closed-form EGN model becomes a main concern in the application of ultra-wideband optical communication systems. In this work, we have investigated the accuracy of the closed-form EGN model for ultra-wideband optical fiber communication systems, where the performance of the system using electronic dispersion compensation, multi-channel nonlinearity compensation and full-field nonlinearity compensation has been evaluated in terms of symbol rate, number of channels and signal power. Our work will provide an insight on the application of the EGN model in next-generation ultra-wideband long-haul optical fiber communication networks.
\end{abstract}

Keywords: Optical fiber communicaiton, EGN model, Ultra-wideband system, Fiber nonlinearities, Digital nonlinearity compensation

\section{INTRODUCTION}

Currently, over $95 \%$ of digital data traffic is carried over optical fiber networks. With the development of technology, people put forward higher requirements on the amount data transmission. To transmit more data at the same time, the symbol transmission rate and communicaiton bandwidth are constantly increasing. As a result, higher and higher spectrum efficiency leads to more serious nonlinearity interference. It is particularly important to accurately and quickly evaluate the signal-to-noise ratio (SNR) of high-speed and ultra-wideband optical fiber communication systems. ${ }^{1}$ The process of optical signal transmission in fiber is complex and unresolvable, but fortunately, the Enhanced GN-model (EGN model), ${ }^{2-11}$ arising from the Gaussian noise perturbation analysis, can evaluate the system SNR accurately. To simplify the calculation, a closed-form formula is proposed using some mathematical approximation. ${ }^{12}$ In this paper, EGN model will be applied in the closed-form manner. The system will be operated at high symbol rate and larg bandwidth to verify the accuracy of EGN model. The transceiver noise has been added to better judge the actual situation, and EGN model has also been adjusted accordingly. ${ }^{13-16}$ At the end of the work, nonlinear compensation using digital back-propagation (DBP) ${ }^{8,17-23}$ algorithm was also employed to verify EGN model comprehensively.

This paper is arranged as follows. The transmission system and its parameter settings as well as the structure of EGN model are shown in Section 2. Section 3 details the simulation results and analyses. Conclusions will be summarized in Section 4.

Further author information: (Send correspondence to Tianhua $\mathrm{Xu}$ )

Tianhua Xu: E-mail: tianhua.xu@ieee.org

Semiconductor Lasers and Applications XI, edited by Wei Li, Werner H. Hofmann,

Yikai Su, Proc. of SPIE Vol. 11891, 1189106 · C 2021 SPIE

0277-786X $\cdot$ doi: $10.1117 / 12.2601076$

Proc. of SPIE Vol. 11891 1189106-1 


\section{TRANSMISSION SETUP AND EGN MODEL}

\subsection{Optical transmission system}

Figure 1 illustrates the structure of the Nyquist-spaced optical fiber communicaiton simulation system. Data are loaded on optical carriers using quadrature amplitude modulation (QAM) format. The combination of wavelength division multiplexing (WDM) and polarisation division multiplexing (PDM) allows the simultaneous transmission of multiple channels. The signal transmission process in fibers is simulated by numerically solving Manakov equation using split-step Fourier transform method (SSFM), and the step length is set small enough to maintain the simulation accuracy. The erbium-doped fiber amplifier (EDFA) is applied to compensate for the power loss of the optical signal. A coherent receiver is employed to detect the signal at the end of system. Digital signal processing is implemented to perform the electronic dispersion compensation (EDC), DBP, matched filtering and the SNR calculation.

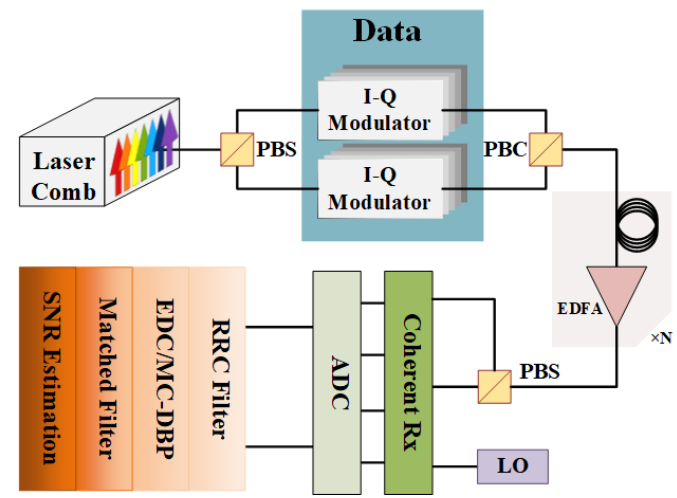

Figure 1. Schematic of the Nyquist-spaced optical fiber communication simulation system using EDC or MC-DBP. PBS: polarisation beam splitter, PBC: polarisation beam combiner, LO: local oscillator, Rx: receiver, ADC: analogue-to-digital convertor

Detailed parameters of the simulation system is listed in Table 1, and the signal is operated at optical launch power unless it is specified.

Table 1. Simulation system parameters.

\begin{tabular}{|l|l|}
\hline Parameter Name & Value \\
\hline Center wavelength & $1550 \mathrm{~nm}$ \\
\hline Attenuation coefficient $(\alpha)$ & $0.2 \mathrm{~dB} / \mathrm{km}$ \\
\hline Chromatic dispersion coefficient $(D)$ & $17 \mathrm{ps} / \mathrm{nm} / \mathrm{km}$ \\
\hline Nonlinear coefficient $(\gamma)$ & $1.2 / \mathrm{W} / \mathrm{km}$ \\
\hline EDFA noise figure & $4.5 \mathrm{~dB}$ \\
\hline TRx noise SNR & $25 \mathrm{~dB}$ \\
\hline Fiber span length & $80 \mathrm{~km}$ \\
\hline Simulation step size & Logarithmic $(\geq 1600$ steps $)$ \\
\hline PMD & 0 \\
\hline
\end{tabular}

\subsection{EGN model}

For dual-polarisation multi-span EDFA amplified Nyquist-spaced WDM transmission systems, the amplifier spontaneous radiation (ASE) noise can be considered as additive Gaussian noise in the EGN assumption. As described in Ref 24, ASE nosie power is expressed as follows: 


$$
\sigma_{\mathrm{ASE}}^{2}=N_{s}(G-1) F_{n} h \mu_{0} R_{s}
$$

where $G$ is the EDFA gain, $F_{n}$ is the EDFA noise figure, $N_{s}$ is the amount of fiber spans, $h$ is Plank constant and $\mu_{0}$ is the center lightwave frequency. For nonlinear noise, the following formula is obtained by Ref $5-7,9,10,15,25-27$ :

$$
\begin{gathered}
\sigma_{\mathrm{NLI}}^{2}=\sigma_{\mathrm{s}-\mathrm{s}}^{2}=N_{s}^{\epsilon+1} \eta_{\mathrm{EGN}} P^{3} \\
\eta_{\mathrm{EGN}} \approx \eta_{\mathrm{GN}}-\frac{80}{81} \frac{\kappa \gamma^{2} L_{\mathrm{eff}}^{2}}{\pi\left|\beta_{2}\right| L_{\mathrm{s}} R_{\mathrm{s}}^{2}}\left[\Phi\left(\frac{N_{\mathrm{ch}}+1}{2}\right)+C+1\right] \\
\eta_{\mathrm{GN}} \approx\left(\frac{2}{3}\right)^{3} \frac{\alpha \gamma^{2} L_{\mathrm{eff}}^{2}}{\pi\left|\beta_{2}\right| R_{\mathrm{s}}^{2}} \operatorname{arsinh}\left(\frac{\pi^{2}}{2}\left|\beta_{2}\right| L_{\mathrm{eff}} N_{\mathrm{ch}}^{2} R_{s}^{2}\right) \\
\epsilon \approx \frac{3}{10} \log _{e}\left[1+\frac{6}{L_{s}} \frac{L_{\mathrm{eff}}}{\operatorname{arsinh}\left(\frac{\pi^{2}}{2}\left|\beta_{2}\right| R_{\mathrm{s}}^{2} L_{\mathrm{eff}} N_{\mathrm{ch}}^{2}\right)}\right]
\end{gathered}
$$

where the constant $\kappa$ is related to the fourth standardised moment (kurtosis) of the input signal constellation. For QPSK (4-QAM), 16-QAM, 64-QAM, and 256-QAM, its values are [1,17/25,13/21,121/200]. Function $\Phi(x)$ is digamma function, and $C \approx 0.557$ is Euler-Mascheroni constant, $\beta_{2}$ is group velocity dispersion coefficient, $\alpha$ is fiber attenuation coefficient $\gamma$ is fiber nonlinear coefficient, $L_{\text {eff }}$ is effective fiber span length, $N_{\mathrm{ch}}$ is the total number of WDM channels, $R_{\mathrm{s}}$ is symbol rate. Considering the combine effect of ASE noise and nonlinear interference, and DBP compensation, ${ }^{20,28}$ EGN model can be written as the following form:

$$
\begin{gathered}
S N R=\frac{P}{\tilde{\kappa} P+\sigma_{\mathrm{ASE}}^{2}+3 \eta\left(\xi_{\mathrm{TRx}} \tilde{\kappa} P+\xi_{\mathrm{ASE}} \sigma_{\mathrm{ASE}}^{2}\right) P^{2}} \\
\xi_{\mathrm{ASE}}=\sum_{n=1}^{N_{\mathrm{s}}} n^{\epsilon+1} \approx \frac{N_{\mathrm{s}}^{\epsilon+1}}{2}+\frac{N_{\mathrm{s}}^{\epsilon+2}}{\epsilon+2} \\
\xi_{\mathrm{TRx}}=N_{\mathrm{s}}^{\epsilon+1} \\
\eta=\eta(\text { WDM bandwidth })-\eta(\mathrm{DBP} \text { bandwidth })
\end{gathered}
$$

where $\tilde{\kappa}$ is a constant related to transceiver noise figure. In this paper, the EGN model results are all derived from Eq. (6).

\section{RESULTS AND DISCUSSIONS}

To investigate the impact of transmission bandwidth, the transmission distance is set as $800 \mathrm{~km}$ (10 spans), and the results are shown in Fig. 2. It can be seen from Fig. 2(a) that the SNR curves of 16QAM, 64QAM and 256QAM are highly coincident, slightly lower than that of QPSK modulation format. A good agreement has been achieved between the numerical simulations and the EGN model. Therefore, to simply the analysis, only 16QAM results are plotted in Fig. 2(b) and 2(c). Observing the distance between EGN model curves and corresponding color markers in Fig. 2(b), the EGN model under ultra-large bandwidth is highly credible. The fitting curves in Fig. 2(c) is plotted to illustrate the accuracy of EGN model in detail. The closed-form EGN model is inaccurate in estimating self-channel interaction (SCI) ${ }^{7}$ effect, since the calculation of estimating SCI effect can not be simplified. Therefore, the performance of EGN model in a small bandwidth is not as accurate as in a large bandwidth, as shown in the shaded area in Fig. 2(c). However, the deviation of EGN model is always within $0.2 \mathrm{~dB}$, which is small enough to be considered as an accurate representation. At a large bandwidth, such as $0.6 \mathrm{THz}$ to $3.3 \mathrm{THz}$, EGN model shows a good accuracy. In summary, in high-speed ultra-wideband optical communication systems, EGN model has sufficient accuracy as a tool to estimate SNR quickly.

The system using multi-channel digital back-propagation (MC-DBP) for compensating nonlinear interaction is also investigated. EGN model results are obtained by solving Eq. (6), where $\eta$ (DBP bandwidth) is calculated 

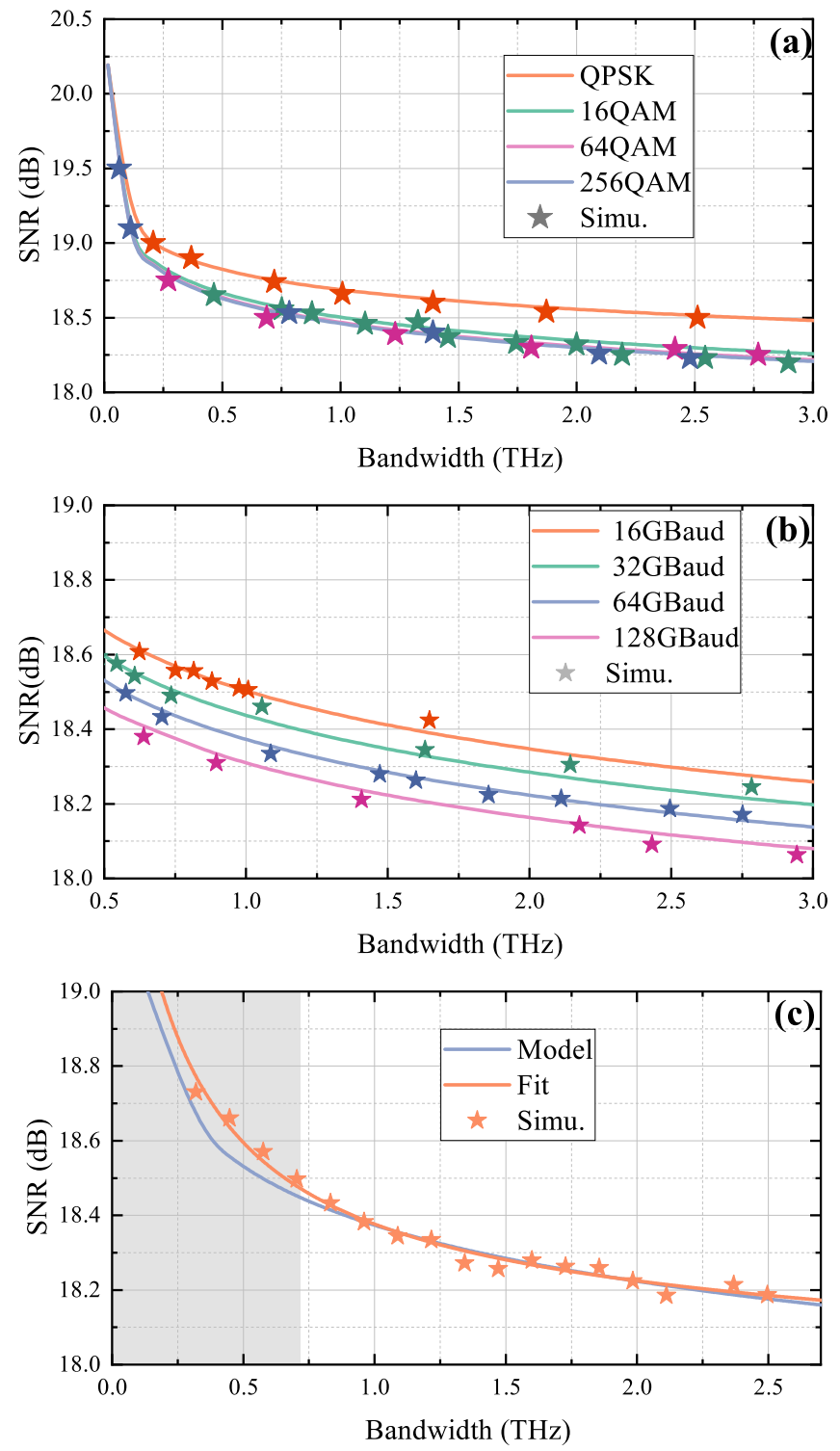

Figure 2. (a): Model v.s. simulation in different modulation formats at 16 GBaud. (b): The accuracy of EGN Model at different symbol rate using 16QAM. (c): Details of Simulation results and EGN model results at 64 GBaud in 16QAM. The blue line is fitted by simulation results. 
by substituting the amount of MC-DBP channels for $N_{\text {ch }}$ in Eq. (3) and Eq. (4). The results are shown in Fig. 3. It can be seen that EGN model matches the simulation results well. In addition, the relationship between SNR and MC-DBP bandwidth is not linear, but close to "S" shape. This is more obvious at high symbol rate (comparing the orange curve and blue curve in Fig. 3). Therefore, the compensation bandwidth should be appropriately selected to obtain the maximum performance-to-cost ratio.

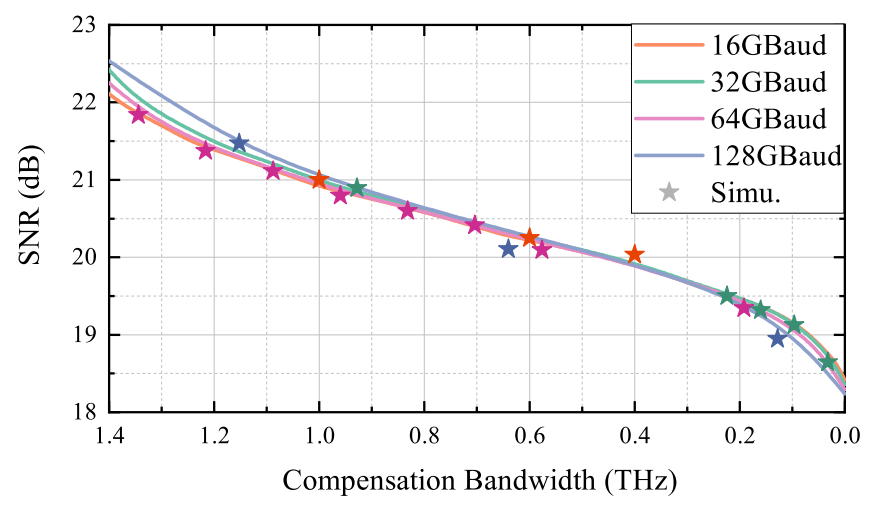

Figure 3. Model v.s. simulation at different nonlinear compensation bandwidth. $0 \mathrm{~Hz}$ means chromatic dispersion compensation only. The simulation system was launched at around $1.5 \mathrm{THz}$ (Round up the decimal part).

The accuracy at different powers of EGN model has been studied using a system with a bandwidth of 1.5 THz to transmit $800 \mathrm{~km}(80 \mathrm{~km} \mathrm{x} \mathrm{10)} \mathrm{in} \mathrm{16-QAM} \mathrm{at} 16$ GBaud and 64 GBaud. Due to the nonlinearity, the system will have an optimal power that maximizes the SNR. For EGN model, the premise is Gaussian noise assumption, which requires nonlinear effect cannot be too strong. So when the power gets larger and larger, the accuracy of the EGN model will decrease. However from a practical engineering perspective, the system will be launched near the optimal power, so it is not of high significance to study the accuracy of the model under strong nonlinearity. As shown in Fig. 4, EGN model is accurate enough near the optimal power.

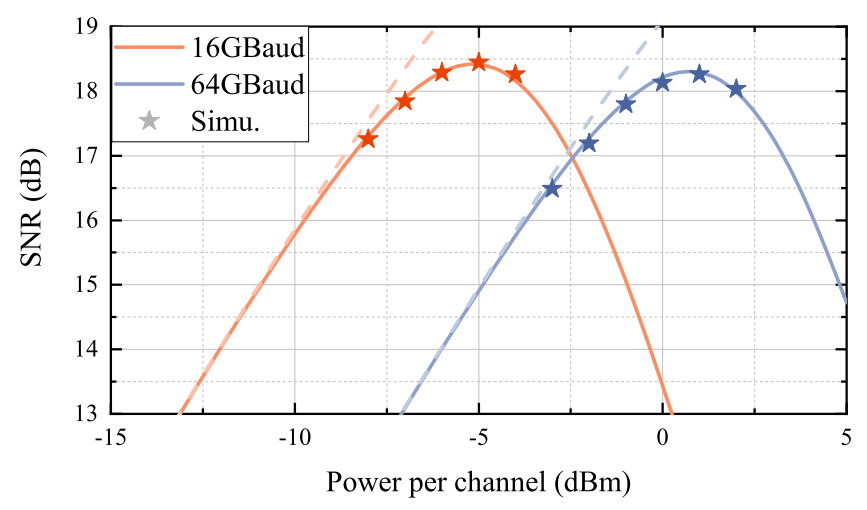

Figure 4. Model v.s. simulation at different launched power. Dotted lines is plotted when only ASE noise exists.

\section{CONCLUSIONS}

In this paper, we have investigated the accuracy of the closed-form EGN model under different transmission scenarios. As a result, the EGN model shows extremely high accuracy at ultra-large bandwidth to estimate optical communication system's SNR quickly. For systems with nonlinear compensation methods (MC-DBP), the EGN model still has a high credibility. We extended the conclusions in the original paper ${ }^{2,7}$ at larger bandwidth and higher symbol rate, and proved the accuracy of the EGN model in more extreme cases. In summary, the closed-form EGN model can quickly and accurately evaluate the high-speed ultra-wideband optical 
communication system's SNR, near the optimal power with and without the use of MC-DBP to compensate for nonlinear interference.

\section{ACKNOWLEDGMENTS}

This work is supported by EU Horizon 2020 Grant 101008280 and Tianjin University Independent Innovation Fund (2021XYK-0004).

\section{REFERENCES}

[1] Bononi, A., Dar, R., Secondini, M., Serena, P., and Poggiolini, P., [Fiber Nonlinearity and Optical System Performance], 287-351, Springer International Publishing, Cham (2020).

[2] Poggiolini, P., Bosco, G., Carena, A., Curri, V., Jiang, Y., and Forghieri, F., "The GN-model of fiber non-linear propagation and its applications," Journal of Lightwave Technology 32(4), 694-721 (2014).

[3] Poggiolini, P., "The GN model of non-linear propagation in uncompensated coherent optical systems," Journal of Lightwave Technology 30, 3857-3879 (Dec 2012).

[4] Poggiolini, P., Carena, A., Curri, V., Bosco, G., and Forghieri, F., "Analytical modeling of nonlinear propagation in uncompensated optical transmission links," IEEE Photonics Technology Letters 23(11), 742-744 (2011).

[5] Serena, P. and Bononi, A., "On the accuracy of the Gaussian nonlinear model for dispersion-unmanaged coherent links," in [European Conference $\&$ Exhibition on Optical Communication], (2013).

[6] Carena, A., Bosco, G., Curri, V., Jiang, Y., Poggiolini, P., and Forghieri, F., "EGN model of non-linear fiber propagation," Optics Express 22(13), 16335-16362 (2014).

[7] Poggiolini, P., Bosco, G., Carena, A., Curri, V., Jiang, Y., and Forghieri, F., "A simple and effective closedform GN model correction formula accounting for signal non-Gaussian distribution," Journal of Lightwave Technology 33, 459-473 (Jan 2015).

[8] Shevchenko, N. A., Xu, T., Domani, L., Gabriele, L., Ives, D. J., Killey, R. I., and Polina, B., "Modeling of nonlinearity-compensated optical communication systems considering second-order signal-noise interactions," Optics Letters 42(17), 3351- (2017).

[9] Zefreh, M. R., Carena, A., Forghieri, F., Piciaccia, S., and Poggiolini, P., "A GN/EGN-model real-time closed-form formula tested over 7,000 virtual links," (2019).

[10] Poggiolini, P., "A generalized GN-model closed-form formula," (2018).

[11] Semrau, D., Sillekens, E., Bayvel, P., and Killey, R. I., "Modeling and mitigation of fiber nonlinearity in wideband optical signal transmission [Invited]," Journal of Optical Communications and Networking 12, C68-C76 (Jun 2020).

[12] Bosco, G., Carena, A., Zefreh, M. R., Poggiolini, P., and Forghieri, F., "Advances in modeling and mitigation of nonlinear effects in uncompensated coherent optical transmission systems," in [2020 European Conference on Optical Communications (ECOC)], 1-4 (2020).

[13] Xu, T., Jacobsen, G., Popov, S., Li, J., Vanin, E., Wang, K., Friberg, A. T., and Zhang, Y., "Chromatic dispersion compensation in coherent transmission system using digital filters," Optics Express 18, 1624316257 (Jul 2010).

[14] Liga, G., Xu, T., Alvarado, A., Killey, R. I., and Bayvel, P., "On the performance of multichannel digital backpropagation in high-capacity long-haul optical transmission," Optics Express 22, 30053-30062 (Dec 2014).

[15] Xu, T., Shevchenko, N. A., Lavery, D., Semrau, D., Liga, G., Alvarado, A., Killey, R. I., and Bayvel, P., "Modulation format dependence of digital nonlinearity compensation performance in optical fibre communication systems," Optics Express 25, 3311-3326 (Feb 2017).

[16] Semrau, D., Lavery, D., Galdino, L., Killey, R. I., and Bayvel, P., "The impact of transceiver noise on digital nonlinearity compensation," Journal of Lightwave Technology 36(3), 695-702 (2018).

[17] Ip, E. and Kahn, J. M., "Compensation of dispersion and nonlinear impairments using digital backpropagation," Journal of Lightwave Technology 26, 3416-3425 (Oct 2008). 
[18] Rafique, D., "Fiber nonlinearity compensation: Commercial applications and complexity analysis," Journal of Lightwave Technology 34, 544-553 (Jan 2016).

[19] Lin, C.-Y., Holtmannspoetter, M., Asif, M. R., and Schmauss, B., "Compensation of transmission impairments by digital backward propagation for different link designs," in [36th European Conference and Exhibition on Optical Communication], 1-3 (2010).

[20] Tanimura, T., Nölle, M., Fischer, J. K., and Schubert, C., "Analytical results on back propagation nonlinear compensator with coherent detection," Optics Express 20, 28779-28785 (Dec 2012).

[21] Rafique, D. and Ellis, A. D., "Impact of signal-ASE four-wave mixing on the effectiveness of digital backpropagation in 112 Gb/s PM-QPSK systems," Optics Express 19(4), 3449-54 (2011).

[22] Beygi, L., Irukulapati, N. V., Agrell, E., Johannisson, P., Karlsson, M., Wymeersch, H., Serena, P., and Bononi, A., "On nonlinearly-induced noise in single-channel optical links with digital backpropagation," Optics Express 21(22), 26376-26386 (2013).

[23] Galdino, L., Semrau, D., Lavery, D., Saavedra, G., Czegledi, C. B., Agrell, E., Killey, R. I., and Bayvel, P., "On the limits of digital back-propagation in the presence of transceiver noise," Optics Express 25, 4564-4578 (Feb 2017).

[24] Agrawal and G., P., [Fiber-optic communication systems], Wiley, (2004).

[25] Xu, T., Shevchenko, N. A., Zhang, Y., Jin, C., Zhao, J., and Liu, T., "Information rates in Kerr nonlinearity limited optical fiber communication systems," Optics Express 29, 17428-17439 (May 2021).

[26] Mecozzi, A. and Essiambre, R.-J., "Nonlinear shannon limit in pseudolinear coherent systems," Journal of Lightwave Technology 30(12), 2011-2024 (2012).

[27] Serena, P., Bononi, A., and Rossi, N., "The impact of the modulation dependent nonlinear interference missed by the Gaussian noise model," in [European Conference on Optical Communication], (2014).

[28] Jin, C., Shevchenko, N. A., Li, Z., Popov, S., Chen, Y., and Xu, T., "Nonlinear coherent optical systems in the presence of equalization enhanced phase noise," Journal of Lightwave Technology 39(14), 4646-4653 (2021). 